\title{
Fractal Dynamics
}

\author{
Raf Dekeyser and Andrzej Komoda* \\ Instituut voor Theoretische Fysica, Katholieke Universiteit Leuven, B-3030 Leuven, Belgium
}

Amos Maritan ${ }^{+}$

Dipartimento di Fisica dell' Universitá degli Studi di Bari, I-70126 Bari, Italy

and

Attilio Stella ${ }^{\ddagger}$

Dipartimento di Fisica "Galileo Galilei" dell' Universitá degli Studi di Padova, I-35131 Padova, Italy

Received March 7, 1989; accepted April 7, 1989

\begin{abstract}
An overview is given of the known relations between various dynamical properties of fractal structure, such as vibration density, diffusion and electrical resistivity. Examples are given from the family of walk-generated fractals: SAW with and without bridges, $k$-tolerant walks and random walks. Results are also discussed for a deterministic model, depending on a topological parameter $f$ which describes the relative scaling between the sizes of clusters and bridges in the fractal.
\end{abstract}

\section{Introduction}

Static (geometric) properties of fractals have been widely accepted as describing properties of a large collection of physical objects that exhibit some kind of self-similarity [1]. One of the first examples was given by the use of the selfavoiding walk (SAW) as a model for describing the relation between the mass and the linear size of a polymer [2, 3]. Although materials like alloys may be better described by the fractals appearing in the percolation problem, the properties of polymeric substances will be best understood in terms of fractal structures obtained through some kind of linear walk. The conformations of these walks may be determined by various types of interactions and their statistics may become temperature dependent. This is, e.g., the case for polymers undergoing a $\Theta$-transition to the collapsed state [4].

During recent years, a lot of attention has been devoted to the dynamical properties of fractals. This was triggered by some experiments in which the energy density of the vibrational states of the fractal object played an important role. These excitations were called "fractons" by Alexander and Orbach [5], who also pointed out the relation between these fractons and other dynamical properties. Most theoretical and numerical work up to now concentrated on the properties of percolation clusters and related models. The purpose of the present report is to show that also walk generated structures are interesting and challenging enough and that their investigation certainly contributes substantially to our understanding of fractal dynamics.

- Present address: Limburgs Universitair Centrum, B-3610 Diepenbeek, Belgium.

† Also at Istituto Nazionale di Fisica Nucleare, Sezione di Bari, Italy.

‡ Also at International School for Advanced Studies, I-34014 Trieste, Italy and Unitá di Padova, Gruppo Nazionale di Struttura della Materia del Consiglio Nazionale delle Ricerche e Centro Interuniversitario di Struttura della Materia, Padova, Italy.
In this paper we first briefly review the basic concepts in this domain and the interconnection between various dynamical phenomena; thereafter we will report some results obtained both by numerical and analytical methods, on random and on special deterministic fractal models respectively.

\section{Dynamical properties of fractals}

The relation between various dynamical properties, like harmonic vibrations and diffusion, was first described for systems with random dynamical parameters [6], but applies equally to systems with structural randomness, as they occur in many fractals. These properties are described by equations of the general form

$\Delta u(\boldsymbol{r}, t)=T u(\boldsymbol{r}, t)$

where $T$ is a differential operator with respect to the time $t$. For the diffusion equation, $T=\partial / \partial t$ and $u$ represents the probability $\boldsymbol{P}$ for finding a particle at site $\boldsymbol{r}$. For the vibrations $T=\partial^{2} / \partial t^{2}$ and $u$ describes the local deformation from equilibrium. On discrete lattice systems, $\Delta$ has to be approximated by

$\Delta u(r) \approx \Delta^{\prime} u_{i} \propto \sum_{j}\left(u_{j}-u_{i}\right)$,

where $j$ runs over the nearest neighbours of site $i$.

The solutions of eq. (1.1) may be obtained from the eigenfunctions of the $\Delta^{\prime}$-operator (with suitable boundary conditions). They obey

$\left(\Delta^{\prime}+E\right) \Psi_{E}(\boldsymbol{r})=0$.

The vibrations are, e.g., described with the amplitudes $c_{E}$ by

$u(\boldsymbol{r}, t)=\sum_{E} c_{E} \Psi_{E}(\boldsymbol{r}) \exp (-\mathrm{i} t \sqrt{E})$

The diffusion equation, with the initial condition $P(r, 0)=$ $\delta_{r, 0}$ is solved as

$$
\begin{aligned}
P(\boldsymbol{r}, t) & =\sum_{E} \psi_{E}^{*}(0) \Psi_{E}(\boldsymbol{r}) \mathrm{e}^{-E t} \\
& \approx \int \mathrm{d} E \varrho(E) \Psi_{E}^{*}(0) \Psi_{E}(\boldsymbol{r}) \mathrm{e}^{-E t}
\end{aligned}
$$

Here, $\varrho(E)$ is the spectral density of the $\Delta^{\prime}$. The long-time behaviour of $P(r, t)$ is dominated by the lower part of this spectrum. If, in analogy with the vibrational spectrum in 
Euclidean spaces, we define a spectral dimension $d$ by

$\tilde{\varrho}(\omega)=\varrho(E) \frac{\mathrm{d} E}{\mathrm{~d} \omega} \propto \omega^{d-1}$

(where $E=\omega^{2}$ ), and thus

$\varrho(E) \propto E^{-1+d / 2}$,

it becomes immediately clear from eq. (1.5) that for $t \rightarrow \infty$

$P(0, t) \propto t^{-d / 2}$.

The diffusion of a particle on a fractal structure is usually described in terms of the effective dimensionality $d_{w}$ of the random walk problem, through the relation

$\left\langle r^{2}(t)\right\rangle \propto t^{2 / d_{w}}$,

which links the mean square displacement to the diffusion time. The exponent $d_{\mathrm{w}}$ can be connected to the spectral dimension $d$ through a scaling argument for $P(r, t)$, which should behave like

$P(r, t) \simeq r^{-d} f\left(r^{d_{w}} / t\right)$.

The exponent $d$ is the geometrical fractal dimension of the structure and it appears in eq. (1.10) for reasons of normalization. Since this expression can also be written as

$t^{-d / d_{\mathrm{w}}} g\left(r^{d_{\mathrm{w}}} / t\right)$

[with $g(x)=x^{-d / d_{w}} f(x)$ ], comparison with eq. (1.8) tells us that

$\tilde{d}=\frac{2 \bar{d}}{d_{\mathrm{w}}}$.

This equation allows us to determine the low frequency scaling of the spectral density of the vibrations through the asymptotic behaviour of the diffusion for $t \rightarrow \infty$.

It was soon discovered that the previously defined exponents are also related to the problem of the electrical resistivity on a fractal resistor network. If the resistance $\Omega(r)$ between two points at a distance $r$ on the fractal (with identical resistors on the bonds of the structure) scales for $r \rightarrow \infty$ like

$\Omega(r) \propto r^{\zeta}$,

one can prove [7] that

$d_{\mathrm{w}}=d+\zeta$.

Since this connection was first established through Einstein's relation

$\sigma=\frac{e^{2} \varrho}{k T} D$

between the electrical conductivity $\sigma$, density $\varrho$ and diffusion constant $D$, relation (1.14) is often called the Einstein relation for fractals. It can also be derived from the Green function of the $\Delta^{\prime}$-operator from eq. (1.3) [8]. This relation provides us with an independent way of determining the dynamical exponents, through the direct calculation of the exponent $\zeta$.

\section{Fractons on random walks}

A lot of attention in the literature has been spent on the dynamical properties of percolation clusters at criticality (for a recent review, see Ref. [9]). The best known result is the
Alexander-Orbach conjecture [5] that for such fractals $d=4 / 3$ in all dimensions; this conjecture has proven to be a very good approximation. We will nevertheless focus our discussion mainly on random walk problems, not only because our own contribution was in this field, but also because historically the whole discussion started when Stapleton et al. [10] explained their experiments on proteins by assuming a SAW-like fractal nature, with a spectral density (1.6), where they conjectured $\tilde{d}=\bar{d}_{\mathrm{SAW}}(=5 / 3)$. Since a SAW is topologically a one-dimensional structure, both vibrations and diffusion on it should be identical to the $d=1$ problem. This means, e.g., that for vibrations eq. (1.6) is valid with $d=1$. For diffusion, we can say that the average length traveled along the SAW after $t$ steps will be proportional to $\sqrt{t}$, which corresponds to a real distance $\langle r\rangle \propto t^{1 / 2 d}$, or $d_{\mathrm{w}}=2 \bar{d}$; from eq. (1.12), we again obtain $d=1$. The resistivity, finally, tells us that two points at a distance $r$ will on the average have an $r^{d}$ length along the SAW between them; thus $\zeta=\bar{d}$ and from eq. (1.14) again $d_{\mathrm{w}}=2 \bar{d}$.

It has been suggested [11] that the above results might change drastically when - after construction of the SAW walk - one enriches the structure by adding bonds (either for elastic forces, diffusion paths or resistors) between all visited nearest-neighbour sites of the embedding lattice. The conjecture was based on the remark that this might form a sufficiently compact structure, on which the diffusion would be normal, i.e., $d_{\mathrm{w}}=2$, such that $d=d$. Through extensive numerical analysis [12], however, we have established that this does not happen, at least not for a simple SAW with the addition of all possible nearest-neighbour bridges. (This does, of course, not exclude the possibility that such a phenomenon might occur in a more collapsed state, which would arise from a SAW with strong interactions.) Even if $d_{\mathrm{w}}=2$ is clearly excluded, there has been much debate in the recent literature on whether $d=1$ and $\zeta=d$ strictly apply to the SAW with nearest neighbour bridges. Many authors claimed small deviations from these values, both on the basis of Monte Carlo and other theoretical evidence. However, a recent systematic approach to the conduction and topological properties of the SAW with bridges [13] clearly showed that $\zeta \approx 1.33$, excluding small deviations and confirming the previous conjectures [12]. Since a nearest-neighbour bridge is, on a coarse-grained level, equivalent with a self-intersection of the walk, we have also investigated the so-called $k$-tolerant walks, in which each lattice site can be visited at most $k$ times. It was established before [14] that $k$-tolerant walks belong to the same universality class as the SAW, as far as the static fractal properties are concerned. Through a direct numerical study of the diffusion problem on 2-tolerant walks, we also established that this universality is also valid for the dynamical parameters $d_{\mathrm{w}}$ and $d$. Also the SAW with bridges belongs to this same class. The behaviour of the above models is thus very different from that of percolation cluster backbones, for which the linear character is a property gradually disappearing upon consideration of larger and larger scales, where multiple connectivity dominates the dynamic properties.

In the limit $k \rightarrow \infty$, the $k$-tolerant walk becomes a free random walk (RW), which might be a good model for long polymers without strong repulsive forces between its constituents. The mathematical properties of a RW are very well established [15], and it is well known that $d=2$ for $d \geqslant 2$. The electrical resistivity was the first dynamical property 


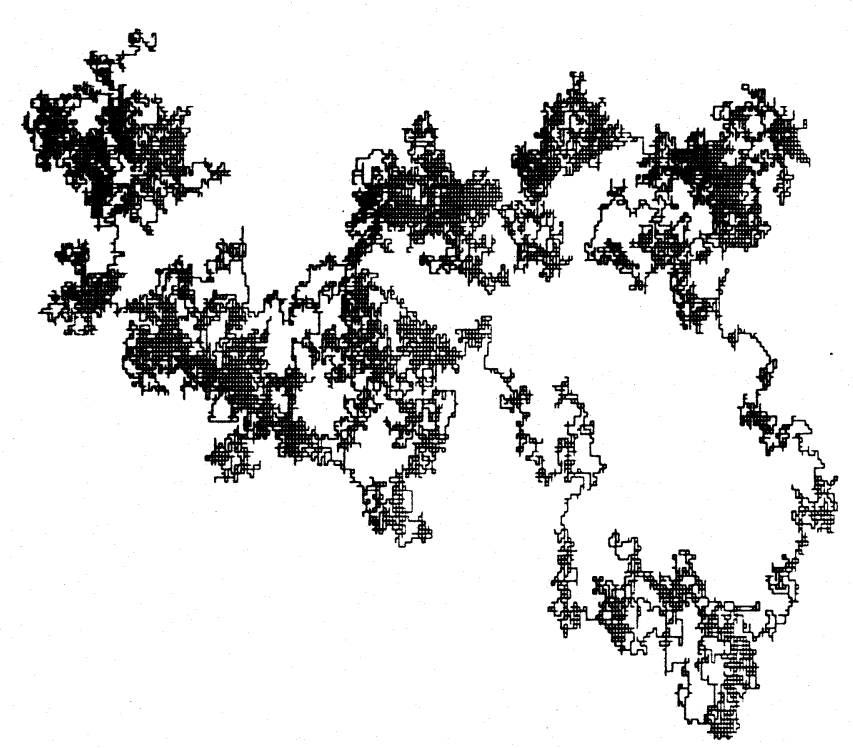

Fig. 1. Typical sample of fractal structure generated by a random walk of 40000 steps on the square lattice.

investigated on the structure generated by the RW on a regular lattice [16] (in this reference, however, the resistivity of a bond was equal to the number of times this bond was visited by the RW; the general belief is that this model is in the same universality class as a model with unit resistances for all visited bonds, such as was investigated by us). Through Monte-Carlo methods, it was found that $\zeta \approx 1$ and 1.5 for $d=2$ and 3 respectively. Through the relations (1.12) and (1.14) this leads to $d=4 / 3$ and $8 / 7$ respectively. In $d=3$, the diffusion problem was studied directly [17], confirming the result $d_{\mathrm{w}}=3.5$. In the same letter, however, it was argued that, since an infinite $\mathrm{RW}$ in $d=2$ occupies the entire space, the dynamical properties of the generated structure must be identical with those of an Euclidean lattice, leading to $d_{\mathrm{w}}=2, \delta=2$ and $\zeta=0$.

It is indeed true that a $\mathrm{RW}$ with $L$ steps becomes a trivial object in $d=2$ in the $L \rightarrow \infty$ limit. If $\left\langle r^{2}(L, t)\right\rangle$ is the mean square distance traveled by a diffusing particle at time $t$ on a $\mathrm{RW}$ of length $L$, one clearly should have

$\lim _{t \rightarrow \infty}\left[\lim _{L \rightarrow \infty} \frac{\ln \left\langle r^{2}(L, t)\right\rangle}{\ln t}\right]=1$

but, for all finite $L$

$\lim _{t \rightarrow \infty}\left[\frac{\ln \left\langle r^{2}(L, t)\right\rangle}{\ln t}\right]=0$.

In spite of these statements, there is abundant numerical evidence that for all large, but finite, $L$-values, the RW behaves as a non-trivial self-similar fractal over a large range of intermediate length scales. In Fig. 1 we show a typical sample of such a structure, generated by a 40000 step RW on the square lattice. One should remark how clusters of occupied sites, holes and loops are present in various sizes. The clusters are often connected through long and narrow paths that will hinder the free diffusion of a particle (or the propagation of a phonon). When $L$ increases, the whole pattern will grow, and one can expect relatively more clusters of larger diameter. Such a structure will undoubtedly lead to a situation where the diffusion is initially, for small $t$, rather normal [the particle diffuses inside a large cluster with $d_{\mathrm{w}}=2$ (2.1)], then goes over into an intermediate regime, after which

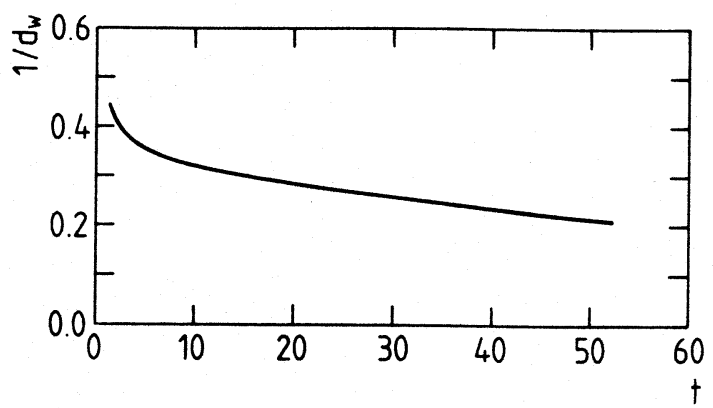

Fig. 2. Estimates for $1 / d_{\mathrm{w}}$ from eq. (2.3) with $\Delta=1$ on a 50 -step random walk on the square lattice.

it very slowly crosses over into the asymptotic region, where for $t \rightarrow \infty$ according to eq. (2.2) one should have $d_{\mathrm{w}}=\infty$.

An extensive numerical analysis in this intermediate region $[14,18]$ seems to indicate the presence of logarithmic corrections to the expression (1.9). If one forgets about these corrections, one may observe the cross-over from the initial normal diffusion into this large intermediate region of fractal diffusion, by plotting as a function of $t$ the estimates for $1 / d_{\omega}$ obtained from

$\frac{1}{d_{\omega}} \simeq \frac{1}{2} \frac{\ln \left\langle R^{2}(t+\Delta)\right\rangle /\left\langle R^{2}(t)\right\rangle}{\ln (t+\Delta) / t}$.

For very short RW-lengths, the fractal structure is too poor to manifest itself, and the curve is monotonously decreasing (see, e.g., Fig. 2). For longer lengths, the cross-over from normal to fractal behaviour can easily be seen (see Fig. 3), but the second cross-over time (due to the finiteness of the structure) is much too large to be observed; obviously the fractal plateau stretches out over a very long time scale. If one keeps the time $t$ fixed at a relatively small value one can also study explicitly the cross-over from fractal to normal behaviour [19]. From a physical point of view, however, we believe that understanding the fractal dynamical behaviour is a more interesting problem.

In order to obtain an independent check on the validity of our conclusion that the global dynamical behaviour of the RW is determined by a spectral dimensionality $d=4 / 3$, we have repeated the electrical resistivity calculations on our RW's, using the transfer-matrix technique [20], and we find confirmation of the results of reference [16] for the longer walks $(\zeta=1.05 \pm 0.05)$, whereas for short walks the estimates for $\zeta$ become smaller. Since the resistivity is a global property of a walk, its behaviour for long walks is indeed dominated by the presence of the intermediate plateau (Fig. 3).

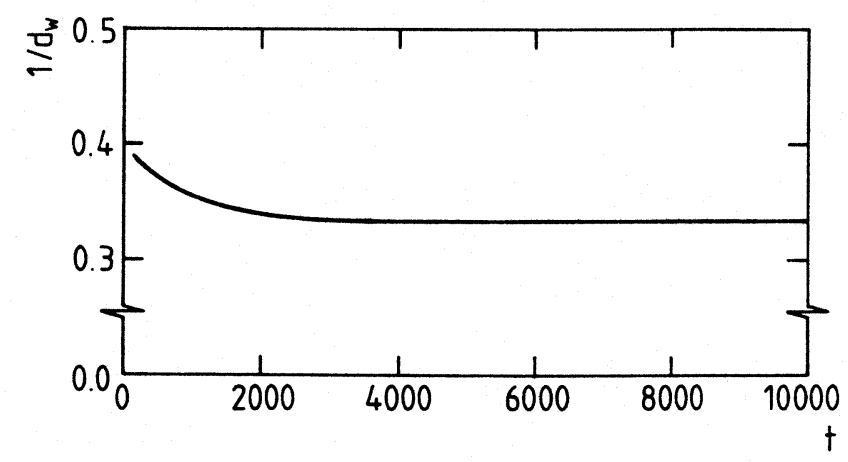

Fig. 3. Estimates for $1 / d_{\mathrm{w}}$ from eq. (2.3) with $\Delta=100$ on a 20000 -step random walk on the square lattice. 


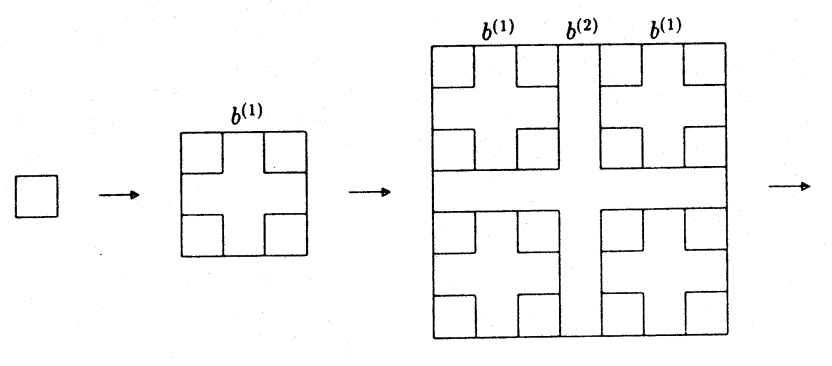

Fig. 4. Iterative construction of a class of models, depending on a family of parameters $\left\{b^{(n)}\right\}$.

\section{A deterministic model}

In order to better understand the results obtained in our numerical simulation, we decided to perform some analytical calculations on deterministic fractals, whose structure tries to reflect the basic properties of the RW fractals. From our simulations (as in Fig. 1) we learned that such fractals contained both clusters and bridges of various sizes. We have combined these in a model, whose infinite structure is obtained iteratively as demonstrated in Fig. 4. The links represented by $b^{(n)}$ may scale in an as yet unspecified manner. The Euclidean distance between the beginning and end points of these $b^{(n)}$-links need not to be constant, as might be suggested by our figure; if these distances, say $l_{n}$ scale like $l_{n} \propto L^{n}$ with $L \leqslant 2$, the fractal dimension $\bar{d}$ of our structure will still be equal to 2 , like in our RW fractals. The path lengths of these links, however, may scale in a different way, since these links may have rather irregular forms. This means that we may, e.g., consider our structure as a model for a fractal resistor network with $\bar{d}=2$, but with resistances $R_{n}$ on the $b^{(n)}$-links that scale as $R_{n} \propto f^{n}$, where $f$ may be any positive number. The same network may be used for diffusion calculations; neglecting the waiting times introduced by the lengths of these links, the analogon of this hierarchy of resistances $R_{n}$ is a hierarchy of diffusion transition rates $b_{n} \propto f^{-n}$. Likewise, for the vibrational problem this corresponds to a hierarchy of spring constants scaling as $f^{-n}$. In principle, we have a different model for every value of $f$.

On this model we have performed two independent calculations [21]. The diffusion equations have been decimated on this self-similar structure, leading to the result that the long-time behaviour of $P(\dot{r}, t)$ is dominated by a $d_{w}$-exponent given by

$$
\begin{aligned}
d_{\mathrm{w}} & =2+\ln f / \ln 2 \quad\left(f \geqslant f_{\mathrm{c}}\right) \\
& =2+\ln f_{\mathrm{c}} / \ln 2 \quad\left(f<f_{\mathrm{c}}\right),
\end{aligned}
$$

where

$f_{\mathrm{c}}=1+\sqrt{2} / 2 \simeq 1.707$.

Also, the electrical resistivity has been calculated in an iterative way, and the result confirms the Einstein relation (1.14) for all values of $f: \zeta=d_{w}-2$.

These results would explain the values obtained from our simulations, if we might infer from Fig. 1 that the path lengths of the links between clusters scale linearly with the diameters of the clusters. This would indeed mean that $f=2$, $d_{\mathrm{w}}=3$ and $\tilde{d}=4 / 3$. Although our model predicts nonuniversal values for the dynamical exponents, one should remark that there is a large universal regime $\left(f<f_{\mathrm{c}}\right.$, which includes, e.g., the case $f=1$ or constant link lengths). For these $f$-values we obtain $d_{\mathrm{w}}=2.66$ and $d=1.44$.

A simple intuitive way to understand the result (3.1) goes as follows. Equation (1.10) implies that for all length rescalings $l$ we have

$P(\boldsymbol{r}, t) \simeq l^{d} P\left(l \boldsymbol{r}, l^{d_{\mathrm{w}}} t\right)$.

For normal diffusion on a regular lattice, we have $d_{\mathrm{w}}=2$, which means that time has to be scaled by $l^{2}$ if length is scaled by $l$. In our deterministic model, where it is natural to choose the iterative rescaling factor $l=2$ for the lengths, the above results mean that time has then to be rescaled with a factor $l^{d_{w}}=l^{2} f$. In other words, the fractal structure imposes an additional rescaling of the time by the factor $f$, which represents the rate with which the diffusion probabilities decrease. Smaller diffusion rates (or long bottleneck links as in Fig. 1) need indeed longer times to produce diffusion. Below a certain limit $f_{c}$, however, the most dominant effect is the simple presence of bottlenecks, and not their strengths. The exact expression for $f_{c}$ can be explained with topological arguments.

The main conclusion from these arguments for the diffusion on random walks is that the $d_{\mathrm{w}}=3$ can be explained from the presence of a hierarchy of clusters interconnected with links whose lengths scale approximately with the diameter of the clusters. This corresponds indeed to $f=2$ in our model. For a rescaling of the lengths with a factor $l$, the time has then to be rescaled by $l^{2}$ for normal diffusion, and again by a factor $l$ for the presence of the links: together $l^{3}$.

\section{References}

1. Mandelbrot, B. B., The Fractal Geometry of Nature, Freeman, San Francisco (1982).

2. Flory, P. J., Principles of Polymer Chemistry, Cornell University Press, Ithaca (1969).

3. de Gennes, P. G., Scaling Concepts in Polymer Physics, Cornell University Press, Ithaca (1979).

4. Seno, F. and Stella, A. L., J. Phys (Paris) 49, 739 (1988); Europhys. Lett. 7, 605 (1988).

5. Alexander, S. and Orbach, R., J. Phys. (Paris) Lett. 43, L625 (1982).

6. Alexander, S., Bernasconi, J., Schneider, W. R. and Orbach, R., Rev. Mod. Phys. 53, 175 (1981).

7. Gefen, Y., Aharony, A. and Alexander, S., Phys. Rev. Lett. 50, 77 (1983).

8. Cates, M. E., J. Phys. (Paris) 46, 1059 (1985).

9. Havlin, S. and Ben-Avraham, D., Adv. Phys. 36, 695 (1987).

10. Stapleton, H. J., Allen, J. P., Flynn, C. P., Stinson, D. G. and Kurtz, S. R., Phys. Rev. Lett. 45, 1456 (1980).

11. Helman, J. S., Coniglio, A. and Tsallis, C., Phys. Rev. Lett. 53, 1195 (1984).

12. Dekeyser, R., Maritan, A. and Stella, A. L., Phys. Rev. A36, 2338 (1987).

13. Seno, F. and Stella, A. L. (to be published)

14. Dekeyser, R., Maritan, A. and Stella, A. L., Phys. Rev. B31, 4659 (1985).

15. Montroll, E. W. and Weiss, G. H., J. Math. Phys. 6, 167 (1965).

16. Banavar, J. R., Harris, A. B. and Koplik, J., Phys. Rev. Lett. 51, 1115 (1983).

17. Havlin, S., Weiss, G. H., Ben-Avraham, D. and Moshovitz, D., J. Phys. A17, L 849 (1984).

18. Dekeyser, R., Maritan, A. and Stella, A. L., Phys. Rev. Lett. 58, 1758 (1987).

19. Mana, S. S., Guttmann, A. J. and Hughes, B. D., Phys. Rev. A39, 4337 (1989).

20. Derrida, B., Zabolitzky, J. G., Vannimenus, J. and Stauffer, D., J. Stat. Phys. 36, 31 (1984).

21. Dekeyser, R., Maritan, A. and Stella, A. L. (to be published) 